\title{
DEGENERATE FLAG VARIETIES AND THE MEDIAN GENOCCHI NUMBERS
}

\author{
EVGENY FEIGIN
}

\begin{abstract}
We study the $\mathbb{G}_{a}^{M}$ degenerations $\mathcal{F}_{\lambda}^{a}$ of the type $A$ flag varieties $\mathcal{F}_{\lambda}$. We describe these degenerations explicitly as subvarieties in the products of Grassmannians. We construct cell decompositions of $\mathcal{F}_{\lambda}^{a}$ and show that for complete flags the number of cells is equal to the normalized median Genocchi numbers $h_{n}$. This leads to a new combinatorial definition of the numbers $h_{n}$. We also compute the Poincaré polynomials of the complete degenerate flag varieties via a natural statistics on the set of Dellac's configurations, similar to the length statistics on the set of permutations. We thus obtain a natural $q$-version of the normalized median Genocchi numbers.
\end{abstract}

\section{Introduction}

Let $\mathfrak{g}=\mathfrak{s l}_{n}, G=S L_{n}$. Fix the Cartan decomposition $\mathfrak{g}=\mathfrak{b} \oplus \mathfrak{n}^{-}$, where $\mathfrak{b}$ is a Borel subalgebra, $\mathfrak{b}=\mathfrak{n} \oplus \mathfrak{h}$. In [Fe3] we considered the degenerate algebra $\mathfrak{g}^{a}=\mathfrak{b} \oplus\left(\mathfrak{n}^{-}\right)^{a}$, where $\left(\mathfrak{n}^{-}\right)^{a}$ is an abelian Lie algebra isomorphic to $\mathfrak{n}^{-}$as a vector space. The corresponding Lie group is a semi-direct product $G^{a}=B \ltimes \mathbb{G}_{a}^{M}$, where $\mathbb{G}_{a}$ is the additive group of the field and $M=\operatorname{dim} \mathfrak{n}$. For a dominant integral weight $\lambda$ let $V_{\lambda}$ be the highest weight $\lambda$ irreducible $\mathfrak{g}$-module with a highest weight vector $v_{\lambda}$. The increasing PBW filtration $F_{\bullet}$ on $V_{\lambda}$ is defined as follows:

$$
F_{0}=\mathbb{C} v_{\lambda}, F_{s+1}=\operatorname{span}\left\{x v: x \in \mathfrak{g}, v \in F_{s}\right\}, s \geq 0
$$

(see [Fe1, Fe2, FFoL1, FFoL2, K2]). The associated graded space $V_{\lambda}^{a}=F_{0} \oplus F_{1} / F_{0} \oplus$ $F_{2} / F_{1} \oplus \cdots$ can be naturally endowed with the structure of a $\mathfrak{g}^{a}$ - and $G^{a}$-module. A degenerate flag variety $\mathcal{F}_{\lambda}^{a}$ is a subvariety in $\mathbb{P}\left(V_{\lambda}^{a}\right)$ defined by $\mathcal{F}_{\lambda}^{a}=\overline{\mathbb{G}_{a}^{M} \cdot \mathbb{C} v_{\lambda}}$. These are the $\mathbb{G}_{a}^{M}$-degenerations of the classical (generalized) flag varieties $\mathcal{F}_{\lambda}$ (see $[\mathrm{A}, \mathrm{AS}, \mathrm{Fe} 3, \mathrm{HT}])$. For example, $\mathcal{F}_{\omega_{d}}^{a} \simeq G r(d, n)$ for all fundamental weights. Recall also that in the classical case (for $\mathfrak{g}=\mathfrak{s l}_{n}$ ) the varieties $\mathcal{F}_{\lambda}=G \cdot \mathbb{C} v_{\lambda} \hookrightarrow \mathbb{P}\left(V_{\lambda}\right)$ are the usual flag varieties (maybe partial). In particular, if $\lambda$ is regular, i.e. $\left(\lambda, \omega_{d}\right)>0$ for all $d$, then $\mathcal{F}_{\lambda}$ is isomorphic to the variety $\mathcal{F}_{n}$ of complete flags in $n$-dimensional space $V$. Fix a basis $v_{1}, \ldots, v_{n}$ of $V$.

For all weights $\lambda, \mu$ there exists an embedding of $G^{a}$-modules $V_{\lambda+\mu}^{a} \hookrightarrow V_{\lambda}^{a} \otimes V_{\mu}^{a}$ sending $v_{\lambda+\mu}$ to $v_{\lambda} \otimes v_{\mu}$ (see [FFoL1, FFoL2]). This induces the embedding of varieties $\mathcal{F}_{\lambda+\mu}^{a} \hookrightarrow \mathcal{F}_{\lambda}^{a} \times \mathcal{F}_{\mu}^{a}$. Thus for any $\lambda$ we obtain an embedding of $\mathcal{F}_{\lambda}^{a}$ into the product of Grassmannians. Our first result is an explicit description of this embedding. We state the theorem here for complete flag varieties $\mathcal{F}_{n}^{a}$. For this we need one more piece of notations. Let $p r_{d}: V \rightarrow V$ be the projection along the space $\mathbb{C} v_{d}$ to the linear span of the vectors $v_{i}, i \neq d$.

\footnotetext{
Received by the editors April 20, 2011.
} 
Theorem 0.1. The image of the embedding of the variety $\mathcal{F}_{n}^{a}$ in the product of Grassmann varieties $\prod_{d=1}^{n-1} G r(d, n)$ is equal to the set of chains of subspaces $\left(V_{1}, \ldots, V_{n-1}\right)$, $V_{d} \in G r(d, n)$ such that

$$
p r_{d+1}\left(V_{d}\right) \hookrightarrow V_{d+1}, \quad 1 \leq d \leq n-2 .
$$

Our next goal is to compute the Poincaré polynomial of $\mathcal{F}_{n}^{a}$. Recall that in the classical case the flag variety $\mathcal{F}_{n}$ can be written as a disjoint union of $n$ ! cells, each cell being associated with a torus fixed point. The fixed points are labeled by permutations from $S_{n}$. The length statistics $\sigma \rightarrow l(\sigma)$ gives the complex dimension of the cells. Therefore, the Poincaré polynomial $P_{\mathcal{F}_{n}}(t)$ of $\mathcal{F}_{n}$ is equal to $P_{\mathcal{F}_{n}}(t)=\sum_{\sigma \in S_{n}} t^{2 l(\sigma)}$.

As an immediate corollary of Theorem 0.1 we obtain that the fixed points of the torus $T \subset G^{a}$ action on $\mathcal{F}_{n}^{a}$ are labeled by the sequences $I^{1}, \ldots, I^{n-1}, I^{d} \subset\{1, \ldots, n\}$, $\# I^{d}=d$, satisfying

$$
I^{d} \backslash\{d+1\} \hookrightarrow I^{d+1}, \quad d=1, \ldots, n-2 .
$$

(Note that this set of sequences has a subset with $I^{d} \hookrightarrow I^{d+1}$, which can be naturally identified with the permutations $S_{n}$ ). Our first task is to compute the number of such fixed points. To this end, recall the normalized median Genocchi numbers $h_{n}$, $n=1,2, \ldots$ (also referred to as the normalized Genocchi numbers of second kind). These numbers have several definitions [De, Du, DR, DZ, G, Kr, Vien] (see Section 3 for a review). Here we give the Dellac definition, which is the earliest one and which fits our construction in the best way.

Consider a rectangle with $n$ columns and $2 n$ rows. It contains $n \times 2 n$ boxes labeled by pairs $(l, j)$, with $l=1, \ldots, n$ and $j=1, \ldots, 2 n$. A Dellac configuration $D$ is a subset of boxes, subject to the following conditions: first, each column contains exactly two boxes from $D$ and each row contains exactly one box from $D$, and, second, if the $(l, j)$-th box is in $D$, then $l \leq j \leq n+l$. Let $D C_{n}$ be the set of such configurations. Then $h_{n}$ is the number of elements in $D C_{n}$. The first several median Genocchi numbers (starting from $h_{1}$ ) are as follows: $1,2,7,38,295$. For instance, the two Dellac configurations for $n=2$ are as follows: (we specify boxes in a configuration by putting fat dots inside)
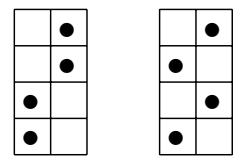

We prove the following theorem:

Theorem 0.2. The number of sequences $I^{1}, \ldots, I^{n-1}$ as above, satisfying (0.1) is equal to $h_{n}$.

We also prove that the Dellac definition [De] is equivalent to the Dumont-Kreweras definition [Du, Kr] (this fact is known to experts [G], [S] but we were unable to find the proof in the literature).

Recall that the length of a permutation $\sigma \in S_{n}$ can be defined as the number of pairs $1 \leq l_{1}<l_{2} \leq n$ satisfying $\sigma\left(l_{1}\right)>\sigma\left(l_{2}\right)$. We define a length $l(D)$ of a Dellac configuration $D$ as the number of squares $\left(l_{1}, j_{1}\right),\left(l_{2}, j_{2}\right) \in D$ such that $l_{1}<l_{2}$ and $j_{1}>j_{2}$. We prove the following theorem:

Theorem 0.3. The Poincaré polynomial $P_{\mathcal{F}_{n}^{a}}(t)$ is given by $\sum_{D \in D C_{n}} t^{2 l(D)}$. 
Our paper is organized in the following way:

In Section 1 we recall main definitions and theorems from [Fe3],

In Section 2 we describe explicitly the image of the embedding of the varieties $\mathcal{F}_{\lambda}^{a}$ into the product of Grassmannians and construct the cell decomposition of $\mathcal{F}_{\lambda}^{a}$.

In Section 3 we study the combinatorics of the median Genocchi numbers and compute the Poincaré polynomials of the complete degenerate flag varieties.

\section{PBW deformation}

1.1. Definitions. We first recall basic definitions and constructions from [FFoL1] and [Fe3]. Let $\mathfrak{g}$ be a simple Lie algebra with the Cartan decomposition $\mathfrak{g}=\mathfrak{n} \oplus \mathfrak{h} \oplus \mathfrak{n}^{-}$. We denote by $M$ the number of positive roots of $\mathfrak{g}$, i.e. $M=\operatorname{dim} \mathfrak{n}$. Let $\mathfrak{b}=\mathfrak{n} \oplus \mathfrak{h}$ be a Borel subalgebra. Then the deformed algebra $\mathfrak{g}^{a}$ is defined as a sum of two subalgebras $\mathfrak{g}^{a}=\mathfrak{b} \oplus\left(\mathfrak{n}^{-}\right)^{a}$, where $\left(\mathfrak{n}^{-}\right)^{a}$ is an abelian Lie algebra isomorphic to $\mathfrak{n}^{-}$ as a vector space. The subalgebra $\left(\mathfrak{n}^{-}\right)^{a} \hookrightarrow \mathfrak{g}^{a}$ is an abelian ideal and the action of $\mathfrak{b}$ on $\left(\mathfrak{n}^{-}\right)^{a}$ is induced from the identification $\left(\mathfrak{n}^{-}\right)^{a} \simeq \mathfrak{g} / \mathfrak{b}$.

Let $G$ be the Lie group of the Lie algebra $\mathfrak{g}$. Let $N, T, N^{-}, B$ be the Lie groups of the Lie algebras $\mathfrak{n}, \mathfrak{h}, \mathfrak{n}^{-}, \mathfrak{b}$. The deformed Lie group $G^{a}$ is defined as a semi-direct product of $B$ and the normal subgroup $\mathbb{G}_{a}^{M}$, where $\mathbb{G}_{a}$ is the additive group of the field (thus $\mathbb{G}_{a}^{M}$ is the Lie group of the Lie algebra $\left(\mathfrak{n}^{-}\right)^{a}$ ). The Borel group $B$ acts on the vector space $\left(\mathfrak{n}^{-}\right)^{a} \simeq \mathfrak{g} / \mathfrak{b}$ via the restriction of the adjoint action and therefore there exists a natural homomorphism from $B$ to $A u t\left(\mathbb{G}_{a}^{M}\right)$, defining the semi-direct product $G^{a}=B \ltimes \mathbb{G}_{a}^{M}$.

For a dominant integral weight $\lambda$ we denote by $V_{\lambda}$ the corresponding irreducible highest weight $\mathfrak{g}$-module with a highest weight vector $v_{\lambda}$. The Lie algebra $\mathfrak{g}^{a}$ and the Lie group $G^{a}$ act on the deformed representations $V_{\lambda}^{a}$, where $\lambda$ are dominant integral weights of $\mathfrak{g}$. The representations $V_{\lambda}^{a}$ are defined as associated graded $g r \cdot V_{\lambda}$ of the representation $V_{\lambda}$ with respect to the $\mathrm{PBW}$ filtration $F_{s}$ :

$$
F_{s}=\operatorname{span}\left\{x_{1} \cdots x_{l} v_{\lambda}: x_{i} \in \mathfrak{g}, l \leq s\right\} .
$$

So $V_{\lambda}^{a}=\bigoplus_{s \geq 0} V_{\lambda}^{a}(s)$, where $V_{\lambda}^{a}(0)=\mathbb{C} v_{\lambda}$ and $V_{\lambda}^{a}(s)=F_{s} / F_{s-1}$ for $s>0$. It is easy to see that the action of $\mathfrak{n}^{-}$on $V_{\lambda}$ becomes abelian on $V_{\lambda}^{a}$ (i.e. it induces the action of $\left.\left(\mathfrak{n}^{-}\right)^{a}\right)$ and the action of the Borel subalgebra induces the action of (the same algebra) $\mathfrak{b}$. The actions of $\left(\mathfrak{n}^{-}\right)^{a}$ and $\mathfrak{b}$ glue together to the action of $\mathfrak{g}^{a}$.

Remark 1.1. Let $\tilde{\mathfrak{g}}^{a}=\mathfrak{g}^{a} \oplus \mathbb{C} p$ be the central, extension of $\mathfrak{g}^{a}$ with a single element $p$ subject to the relations $[p, \mathfrak{b}]=0,\left[p, f_{\alpha}\right]=f_{\alpha}$ for any positive root $\alpha$ and the corresponding weight element $f_{\alpha} \in\left(\mathfrak{n}^{-}\right)^{a}$. Thus the Cartan subalgebra of $\tilde{\mathfrak{g}}^{a}$ has one extra dimension. We note that the $\mathfrak{g}^{a}$-module structure of $V_{\lambda}^{a}$ naturally lifts to the structure of representation of $\tilde{\mathfrak{g}}^{a}$ by setting $p v_{\lambda}=0$ (in general, $\left.p\right|_{V_{\lambda}^{a}(s)}=s$ ). An eigenvalue of the operator $p$ is sometimes referred to as a PBW degree. The character of $V_{\lambda}^{a}$ with respect to $\mathfrak{h} \oplus \mathbb{C} p$ was computed in [FFoL1] for $\mathfrak{s l}_{n}$ and in [FFoL2] for symplectic Lie algebras. We denote the Lie group of $\tilde{\mathfrak{g}}^{a}$ by $\tilde{G}^{a}$, which differs from $G^{a}$ by an additional $\mathbb{C}^{*}$.

Consider the action of $G^{a}$ on the projective space $\mathbb{P}\left(V_{\lambda}^{a}\right)$. Recall that in the classical situation the (generalized) flag varieties are defined as $\mathcal{F}_{\lambda}=G \cdot \mathbb{C} v_{\lambda} \hookrightarrow \mathbb{P}\left(V_{\lambda}\right)$ (see $[\mathrm{K} 1])$. The degenerate flag varieties $\mathcal{F}_{\lambda}^{a} \hookrightarrow \mathbb{P}\left(V_{\lambda}^{a}\right)$ are defined as the closures of the $G^{a}$ 
orbit (or, equivalently, of the $\mathbb{G}_{a}^{M}$ orbit) of the line $\mathbb{C} v_{\lambda}$. We note that in the classical case the orbit $G \cdot \mathbb{C} v_{\lambda}$ already covers the whole flag variety. This is not true in the degenerate case: the orbit $G^{a} \cdot \mathbb{C} v_{\lambda}$ is an affine cell, whose closure gives a projective singular variety $\mathcal{F}_{\lambda}^{a}$.

1.2. The type $A$ case. From now on we assume that $\mathfrak{g}=\mathfrak{s l}_{n}$ and $G=S L_{n}$. Then all positive roots are of the form

$$
\alpha_{i, j}=\alpha_{i}+\cdots+\alpha_{j}, 1 \leq i \leq j \leq n-1
$$

(for instance, $\alpha_{i, i}=\alpha_{i}$ are the simple roots). We denote by $f_{i, j}=f_{\alpha_{i, j}} \in \mathfrak{n}^{-}$and $e_{i, j}=e_{\alpha_{i, j}} \in \mathfrak{n}$ the corresponding root elements. We have $\mathcal{F}_{\omega_{d}}^{a} \simeq \mathcal{F}_{\omega_{d}} \simeq G r(d, n)$. The reason why the degenerate flag varieties are isomorphic to the non-degenerate ones for fundamental weights is that the radicals in $\mathfrak{s l}_{n}$, corresponding to $\omega_{d}$, are abelian. In other words, define the set of positive roots

$$
R_{d}=\left\{\alpha_{i, j}: 1 \leq i \leq d \leq j \leq n-1\right\} .
$$

Define the subalgebra $\mathfrak{u}_{d}^{-}=\operatorname{span}\left\{f_{\alpha}: \alpha \in R_{d}\right\}$. Then $\mathfrak{u}_{d}^{-}$is abelian and $V_{\omega_{d}}=$ $U\left(\mathfrak{u}_{d}^{-}\right) \cdot v_{\lambda}$.

Remark 1.2. Let us explain the difference between the structure of $\mathfrak{g}$-module on $V_{\omega_{d}}$ and the structure of $\mathfrak{g}^{a}$-module on $V_{\omega_{d}}^{a}$. The operators $f_{\alpha}$ act trivially on $V_{\omega_{d}}^{a}$ unless $\alpha \in R_{d}$. Also, $e_{\alpha}$ act trivially on $V_{\omega_{d}}^{a}$ if $\alpha \in R_{d}$. Therefore, $\mathfrak{g}^{a}$ acts on $V_{\omega_{d}}^{a}$ via the projection to the subalgebra

$$
\mathfrak{g}_{d}^{a}=\mathfrak{u}_{d}^{-} \oplus \mathfrak{h} \oplus \operatorname{span}\left\{e_{\alpha}: \alpha \notin R_{d}\right\} .
$$

Similarly, the group $G^{a}$ acts on $G r(d, n)$ via the surjection to the Lie group of $\mathfrak{g}_{d}^{a}$. In particular, the group $G^{a}$ does not act transitively on the deformed flag varieties even in the case of Grassmannians.

Remark 1.3. We note that though $\mathcal{F}_{\omega_{d}}^{a} \simeq \mathcal{F}_{\omega_{d}} \simeq G r(d, n)$, the actions of the Borel groups $B \subset G$ and $B \subset G^{a}$ are very different. Let us consider the case $G=S L_{2}$. Then $\mathfrak{g}^{a}$ is spanned by three elements $e^{a}, h^{a}$ and $f^{a}$ subject to the relations

$$
\left[h^{a}, e^{a}\right]=2 e^{a},\left[h^{a}, f^{a}\right]=-2 f^{a},\left[e^{a}, f^{a}\right]=0 .
$$

Let $\lambda$ be a dominant weight of $\mathfrak{s l}_{2}, \lambda \in \mathbb{Z}_{\geq 0}$. Then $V_{\lambda}^{a}$ is the direct sum of onedimensional subspaces spanned by vectors $v_{l}, l=\lambda, \lambda-2, \ldots,-\lambda$ such that

$$
h^{a} v_{l}=l v_{l}, f^{a} v_{l}=v_{l-2}, e^{a} v_{l}=0 .
$$

Therefore, the Borel subgroup $B$ acts trivially on $\mathcal{F}_{\lambda}^{a} \simeq \mathbb{P}^{1}$. For instance, there exists one point of $\mathbb{P}^{1}$, which is fixed by the action of the whole group $G^{a}$.

Let us now recall the Plücker relations for $\mathcal{F}_{\lambda}[\mathrm{Fu}]$ and the deformed Plücker relations for $\mathcal{F}_{\lambda}^{a}[\mathrm{Fe} 3]$.

Let $1 \leq d_{1}<\cdots<d_{s} \leq n-1$ be a sequence of increasing numbers. Then for any positive integers $a_{1}, \ldots, a_{s}$ the variety $\mathcal{F}_{a_{1} \omega_{d_{1}}+\cdots+a_{s} \omega_{d_{s}}}$ is isomorphic to the partial flag variety

$$
\mathcal{F}\left(d_{1}, \ldots, d_{s}\right)=\left\{V_{1} \hookrightarrow V_{2} \hookrightarrow \cdots \hookrightarrow V_{s} \hookrightarrow \mathbb{C}^{n}: \operatorname{dim} V_{i}=d_{i}\right\}
$$


In particular, if $s=1$, then $\mathcal{F}(d)$ is the Grassmannian $G r(d, n)$ and for $s=n-1$ $\mathcal{F}(1, \ldots, n-1)$ is the variety of the complete flags. We recall that

$$
V_{\omega_{d}}=\Lambda^{d}\left(V_{\omega_{1}}\right)=\Lambda^{d}\left(\mathbb{C}^{n}\right)
$$

and the embedding $\operatorname{Gr}(d, n) \hookrightarrow \mathbb{P}\left(\Lambda^{d} V_{\omega_{1}}\right)$ is defined as follows: a subspace with a basis $w_{1}, \ldots, w_{d}$ maps to $\mathbb{C} w_{1} \wedge \cdots \wedge w_{d}$. For general sequence $d_{1}, \ldots, d_{s}$ one has embeddings:

$$
\mathcal{F}\left(d_{1}, \ldots, d_{s}\right) \hookrightarrow G r\left(d_{1}, n\right) \times \cdots \times G r\left(d_{s}, n\right) \hookrightarrow \mathbb{P}\left(V_{\omega_{d_{1}}}\right) \times \cdots \times \mathbb{P}\left(V_{\omega_{d_{s}}}\right) .
$$

The composition of these embeddings is called the Plücker embedding. The image is described explicitly in terms of Plücker relations. Namely, let $v_{1}, \ldots, v_{n}$ be a basis of $\mathbb{C}^{n}=V_{\omega_{1}}$. Then one gets a basis $v_{J}$ of $V_{\omega_{d}} v_{J}=v_{j_{1}} \wedge \cdots \wedge v_{j_{d}}$ labeled by sequences $J=\left(1 \leq j_{1}<j_{2}<\cdots<j_{d} \leq n\right)$. Let $X_{J} \in V_{\omega_{d}}^{*}$ be the dual basis. We denote by the same symbols the coordinates of a vector $v \in V_{\omega_{d}}: X_{J}=X_{J}(v)$. The image of the embedding

$$
\mathcal{F}\left(d_{1}, \ldots, d_{s}\right) \hookrightarrow \times_{i=1}^{s} \mathbb{P}\left(V_{\omega_{d_{i}}}\right)
$$

is defined by the Plücker relations. These relations are labeled by a pair of numbers $p \geq q, p, q \in\left\{d_{1}, \ldots, d_{s}\right\}$, by a number $k, 1 \leq k \leq q$ and by a pair of sequences $L=\left(l_{1}, \ldots, l_{p}\right), J=\left(j_{1}, \ldots, j_{q}\right), 1 \leq l_{\alpha}, j_{\beta} \leq n$. The corresponding relation is denoted by $R_{L, J}^{k}$ and is given by

$$
R_{L, J}^{k}=X_{L} X_{J}-\sum_{1 \leq r_{1}<\cdots<r_{k} \leq p} X_{L^{\prime}} X_{J^{\prime}}
$$

where $L^{\prime}, J^{\prime}$ are obtained from $L, J$ by interchanging $k$-tuples $\left(l_{r_{1}}, \ldots, l_{r_{k}}\right)$ and $\left(j_{1}, \ldots, j_{k}\right)$ in $L$ and $J$ respectively, i.e.

$$
\begin{gathered}
J^{\prime}=\left(l_{r_{1}}, \ldots, l_{r_{k}}, j_{k+1}, \ldots, j_{q}\right) \\
L^{\prime}=\left(l_{1}, \ldots, l_{r_{1}-1}, j_{1}, l_{r_{1}+1}, \ldots, l_{r_{2}-1}, j_{2}, \ldots, l_{p}\right) .
\end{gathered}
$$

We note that for any $\sigma \in S_{d}$ the equality

$$
X_{j_{\sigma(1)}, \ldots, j_{\sigma(d)}}=(-1)^{\sigma} X_{j_{1}, \ldots, j_{d}}
$$

is assumed in (1.2). We denote the ideal generated by all $R_{L, J}^{k}$ by $I\left(d_{1}, \ldots, d_{s}\right)$.

We introduce the notation

$$
\mathcal{F}^{a}\left(d_{1}, \ldots, d_{s}\right)=\mathcal{F}_{\omega_{d_{1}}+\cdots+\omega_{d_{s}}}^{a}, 1 \leq d_{1}<\cdots<d_{s}<n .
$$

Definition 1.1. Let $I^{a}\left(d_{1}, \ldots, d_{s}\right)$ be an ideal in the polynomial ring in variables $X_{j_{1}, \ldots, j_{d}}^{a}, d=d_{1}, \ldots, d_{s}, 1 \leq j_{1}<\cdots<j_{d}<n$, generated by the elements $R_{L, J}^{k ; a}$ given below. These elements are labeled by a pair of numbers $p \geq q, p, q \in\left\{d_{1}, \ldots, d_{s}\right\}$, by an integer $k, 1 \leq k \leq q$ and by sequences $L=\left(l_{1}, \ldots, l_{p}\right), J=\left(j_{1}, \ldots, j_{q}\right)$, which are arbitrary subsets of the set $\{1, \ldots, n\}$. The generating elements are given by the formulas

$$
R_{L, J}^{k ; a}=X_{l_{1}, \ldots, l_{p}}^{a} X_{j_{1}, \ldots, j_{q}}^{a}-\sum_{1 \leq r_{1}<\cdots<r_{k} \leq p} X_{l_{1}^{\prime}, \ldots, l_{p}^{\prime}}^{a} X_{j_{1}^{\prime}, \ldots, j_{q}^{\prime}}^{a},
$$

where the terms of $R_{L, J}^{k ; a}$ are the terms of $R_{L, J}^{k}$ (1.2) (with a superscript $a$, to be precise) such that

$$
\left\{l_{r_{1}}, \ldots, l_{r_{k}}\right\} \cap\{q+1, \ldots, p\}=\emptyset
$$


Remark 1.4. The initial term $X_{l_{1}, \ldots, l_{p}}^{a} X_{j_{1}, \ldots, j_{q}}^{a}$ is also subject to the condition (1.4), i.e. it is not present in $R_{L, J}^{k ; a}$ if $\left\{j_{1}, \ldots, j_{k}\right\} \cap\{q+1, \ldots, p\} \neq \emptyset$.

Example 1.1. Let $s=1$. Then $I^{a}(d)=I(d)$, since there are no numbers $l$ such that $d+1 \leq l \leq d$ and thus $R_{L, J}^{k ; a}=R_{L, J}^{k}$ (up to a superscript $a$ in the notations of variables $X_{J}$ ). Hence $\mathcal{F}_{\omega_{d}}^{a} \simeq \mathcal{F}_{\omega_{d}}$.

The following theorem is proved in [Fe3].

Theorem 1.1. The variety $\mathcal{F}^{a}\left(d_{1}, \ldots, d_{s}\right) \hookrightarrow \times_{i=1}^{s} \mathbb{P}\left(\Lambda^{d_{i}} \mathbb{C}^{n}\right)$ is defined by the ideal $I^{a}\left(d_{1}, \ldots, d_{s}\right)$.

Example 1.2. Let $s=2, d_{1}=1, d_{2}=n-1$. Then the classical flag variety $\mathcal{F}(1, n-1)$ is a subvariety in $\mathbb{P}^{n-1} \times \mathbb{P}^{n-1}$ defined by a single relation

$$
\sum_{i=1}^{n}(-1)^{i-1} X_{i} X_{1, \ldots, i-1, i+1, \ldots, n}=0 .
$$

The degenerate variety $\mathcal{F}(1, n-1)$ is also a subvariety in $\mathbb{P}^{n-1} \times \mathbb{P}^{n-1}$, defined by a "degenerate" relation

$$
X_{1}^{a} X_{2, \ldots, n}^{a}+(-1)^{n-1} X_{n}^{a} X_{1, \ldots, n-1}^{a}=0
$$

\section{Cell decomposition}

In this section, we describe explicitly the image of $\mathcal{F}_{\lambda}^{a}$ inside the product of Grassmannians and construct the cell decomposition of the degenerate flag varieties. We start with the case of $\lambda=\omega_{d}$.

2.1. Cell decomposition for Grassmannians. Recall that $\mathcal{F}_{\omega_{d}}^{a} \simeq \mathcal{F}_{\omega_{d}} \simeq G r(d, n)$. Given an increasing tuple $L=\left(l_{1}<\cdots<l_{d}\right)$ we set

$$
p_{L}=\operatorname{span}\left(v_{l_{1}}, \ldots, v_{l_{d}}\right) \in G r(d, n) .
$$

The subspace $p_{L}$ is $T$-invariant. Let $k$ be a number such that $l_{k} \leq d<l_{k+1}$.

Proposition 2.1. The orbit $G^{a} \cdot p_{L}$ is an affine cell and $G r(d, n)$ is the disjoint union of all such cells.

Proof. Recall that $G^{a}$ acts on $G r(d, n)$ via the projection to the Lie group of $\mathfrak{g}_{d}$ (see (1.1)). Therefore the elements of $G^{a} \cdot p_{L}$ are exactly the subspaces of $V$ having a basis $e_{1}, \ldots, e_{d}$ of the form

$$
\begin{gathered}
e_{j}=v_{l_{j}}+\sum_{i=1}^{l_{j}-1} a_{i, j} v_{i}+\sum_{i=d+1}^{n} a_{i, j} v_{i}, j=1, \ldots, k \\
e_{j}=v_{l_{j}}+\sum_{i=d+1}^{l_{j}-1} a_{i, j} v_{i}, j=k+1, \ldots, d .
\end{gathered}
$$

Such elements in $\operatorname{Gr}(d, n)$ obviously form an affine cell and one has a decomposition $G r(d, n)=\sqcup_{L} G^{a} \cdot p_{L}$. 
Remark 2.1. Formulas $(2.1)$ and $(2.2)$ can be combined together as follows. Let $[k]_{+}=k$ if $k>0$ and $[k]_{+}=k+n$ if $k \leq 0$. Then each element of $G^{a} \cdot p_{L}$ has a basis $e_{1}, \ldots, e_{d}$ of the form

$$
e_{j}=v_{l_{j}}+\sum_{i=1}^{\left[l_{j}-d\right]_{+}-1} a_{i, j} v_{\left[l_{j}-i\right]_{+}}
$$

Remark 2.2. The orbit $G^{a} \cdot p_{L}$ can be identified with a certain cell $B \cdot p_{J}$ in the usual cell decomposition of $\operatorname{Gr}(d, n)$. Namely, define $J$ as follows:

$$
J=\left(l_{k+1}-d, l_{k+2}-d, \ldots, l_{d}-d, l_{1}-d+n, l_{2}-d+n, \ldots, l_{k}-d+n\right) .
$$

Then the map

$$
\psi: V \rightarrow V, \psi\left(v_{i}\right)=v_{[i-d]_{+}}, i=1, \ldots, n
$$

sends $G^{a} \cdot p_{L}$ to $B \cdot p_{j}$ (this is clear from the explicit description (2.1), (2.2)).

Example 2.1. Let $n=9, d=4$ and $L=(2,3,6,7)$ (thus $k=2$ ). Then the elements of $G^{a} \cdot p_{L}$ can be identified with the following matrices (the columns of a matrix form a basis of the corresponding subspace):

$$
\left(\begin{array}{llll}
* & * & 0 & 0 \\
1 & 0 & 0 & 0 \\
0 & 1 & 0 & 0 \\
0 & 0 & 0 & 0 \\
* & * & * & * \\
0 & 0 & 1 & 0 \\
0 & 0 & 0 & 1 \\
* & * & 0 & 0 \\
* & * & * & *
\end{array}\right) .
$$

Here $*$ denotes arbitrary entries and hence the number of stars coincides with the dimension of the cell.

2.2. Chains of subspaces. In this section we fix the numbers $d_{1}, \ldots, d_{s}$ and write $\mathcal{F}^{a}$ for $\mathcal{F}^{a}\left(d_{1}, \ldots, d_{s}\right)$. Let $v_{1}, \ldots, v_{n}$ be some basis of $V \simeq \mathbb{C}^{n}$. For $1 \leq i<j \leq n$ we define the projections $p r_{i+1, j}: V \rightarrow V$ by the formula

$$
p r_{i+1, j}\left(\sum_{l=1}^{n} c_{l} v_{l}\right)=\sum_{l=1}^{i} c_{l} v_{l}+\sum_{l=j+1}^{n} c_{l} v_{l} .
$$

The goal of this subsection is to prove the following theorem.

Theorem 2.1. The variety $\mathcal{F}^{a} \hookrightarrow G r\left(d_{1}, n\right) \times \cdots \times G r\left(d_{s}, n\right)$ is formed by all sequences $V_{1}, \ldots, V_{s}, V_{l} \in G r\left(d_{l}, n\right)$ such that for all $1 \leq l<m \leq s$

$$
p r_{d_{l}+1, d_{m}} V_{l} \hookrightarrow V_{m} .
$$

Remark 2.3. It is easy to see that the set of conditions (2.4) is equivalent to the subset with $m=l+1$, i.e. to the set of conditions

$$
p r_{d_{l}+1, d_{l+1}} V_{l} \hookrightarrow V_{l+1}, \quad l=1, \ldots, s-1 .
$$

Lemma 2.1. Let $\left(V_{1}, \ldots, V_{s}\right) \in \mathcal{F}^{a}$. Then conditions (2.4) are satisfied. 
Proof. Let us first look at the big cell $\mathbb{G}_{a}^{M} \cdot \mathbb{C} v_{\lambda} \subset \mathcal{F}^{a}$. Note that the line $\mathbb{C} v_{\lambda}$ is represented by the point

$$
\times_{i=1}^{s} \operatorname{span}\left(v_{1}, \ldots, v_{d_{i}}\right) \in \times_{i=1}^{s} G r\left(d_{i}, n\right) .
$$

Take an element $g=\exp \left(\sum s_{i, j} f_{i, j}\right) \in \mathbb{G}_{a}^{M} \subset G^{a}$. Then one has

$$
g \cdot \operatorname{span}\left(v_{1}, \ldots, v_{d}\right)=\operatorname{span}\left(v_{1}+\sum_{j=d}^{n-1} s_{1, j} v_{j+1}, \ldots, v_{d}+\sum_{j=d}^{n-1} s_{d, j} v_{j+1}\right) .
$$

Therefore conditions (2.4) hold for all points from the big cell of the degenerate flag varieties. Since $\mathcal{F}_{\lambda}^{a}$ is the closure of the big cell, the lemma is proved.

Proposition 2.2. Let $V_{1}, \ldots, V_{s}$ be a set of subspaces of $V$ satisfying (2.4) with $\operatorname{dim} V_{l}=d_{l}$. Then $\left(V_{1}, \ldots, V_{s}\right) \in \mathcal{F}^{a}$.

Proof. We know that the image of the embedding

$$
\mathcal{F}^{a} \hookrightarrow \times_{i=1}^{s} G r\left(d_{i}, n\right) \hookrightarrow \times_{i=1}^{s} \mathbb{P}\left(\Lambda^{d_{i}} V\right)
$$

is defined by the set of relations $R_{J, I}^{k ; a}=0$. Our goal is to prove that (2.4) implies that all the relations $R_{J, I}^{k ; a}$ vanish. Fix a pair $1 \leq l \leq m \leq s$. In what follows we denote the projection $p r_{d_{l}+1, d_{m}}$ simply by $p r$.

Let $\left(V_{1}, \ldots, V_{s}\right)$ be a collection of subspaces satisfying (2.4). Fix tuples $I=$ $\left(i_{1}, \ldots, i_{l}\right)$ and $J=\left(j_{1}, \ldots, j_{m}\right)$ and a number $k$. We prove that the relation $R_{J, I}^{k ; a}$ vanishes on $\left(V_{1}, \ldots, V_{s}\right)$. Without loss of generality we assume that $i_{1}, \ldots, i_{k} \notin$ $\left[d_{l}+1, d_{m}\right]$. We also rearrange the entries of $I$ in such a way that the elements from $I \cap\left[d_{l}+1, d_{m}\right]$ are concentrated at the end of $I$, i.e. there exists a number $b$ such that

$$
i_{1}, \ldots, i_{b} \notin\left[d_{l}+1, d_{m}\right], \quad i_{b+1}, \ldots, i_{l} \in\left[d_{l}+1, d_{m}\right] .
$$

Obviously, $b \geq k$. Let $l-c=\operatorname{dim}\left(\operatorname{ker} p r \cap V_{l}\right)$. We fix a basis $e_{1}, \ldots, e_{l}$ of $V_{l}$ such that pre $_{1}, \ldots$, pre $_{c}$ is a basis of $p r V_{l}$ and $e_{c+1}, \ldots, e_{l}$ form a basis of ker $p r \cap V_{l}$. We denote by $a_{s, t}$ the coefficients of the expansion of $e_{s}$ in terms of $v_{t}$ :

$$
e_{q}=\sum_{r=1}^{l} a_{r, q} v_{r}
$$

The idea of the proof is to use the following decomposition of a Plücker coordinate $X_{I}$ :

$$
X_{I}=\sum_{1 \leq \alpha_{1}<\cdots<\alpha_{l-b} \leq l} \pm a_{i_{b+1}, \alpha_{1}} \ldots a_{i_{l}, \alpha_{l-b}} X_{i_{1}, \ldots, i_{b}} .
$$

Here $X_{i_{1}, \ldots, i_{b}}$ is the $\left(i_{1}, \ldots, i_{b}\right)$-th Plücker coordinate of the vector space $\operatorname{span}\left(e_{\beta_{1}}, \ldots, e_{\beta_{b}}\right)$, where the set of $\beta$ 's is complementary to the set of $\alpha$ 's, i.e.

$$
\left\{\beta_{1}, \ldots, \beta_{b}\right\} \cup\left\{\alpha_{1}, \ldots, \alpha_{l-b}\right\}=\left\{i_{1}, \ldots, i_{l}\right\} .
$$

The decomposition (2.6) induces the decomposition of the relation $R_{J, I}^{k ; a}$, such that each term can be shown to vanish. Note that if $b>c$ then $X_{I}$ vanishes on $V_{l}$. We thus assume that $b \leq c$.

Define the subspace

$$
E_{\beta}=\operatorname{pr}\left(\operatorname{span}\left(e_{\beta_{1}}, \ldots, e_{\beta_{b}}\right)\right)
$$


We know that $E_{\beta} \hookrightarrow V_{m}$. In addition, the coordinates $X_{\left(i_{1}, \ldots, i_{b}\right)}$ of the space $\operatorname{span}\left(e_{\beta_{1}}, \ldots, e_{\beta_{b}}\right)$ coincide with the Plücker coordinates $Y_{\left(i_{1}, \ldots, i_{b}\right)}$ of $E_{\beta}$, because $i_{1}, \ldots, i_{b} \notin\left[d_{l}+1, d_{m}\right]$ (we are using the notations $Y_{I}$ to distinguish between Plücker coordinated of different spaces). Since $E_{\beta} \hookrightarrow V_{m}$, the classical relations $R_{J,\left(i_{1}, \ldots, i_{b}\right)}^{k}$ vanish on the pair $\left(E_{\beta}, V_{m}\right)$. Since

$$
E_{\beta} \hookrightarrow \operatorname{span}\left(v_{1}, \ldots, v_{d_{l}}, v_{d_{m}+1}, \ldots, v_{n}\right)
$$

a Plücker coordinate $Y_{q_{1}, \ldots, q_{b}}$ of $E_{\beta}$ vanishes unless non of the indices $q_{\bullet}$ are between $d_{l}+1$ and $d_{m}$. Hence the degenerate Plücker relation $R_{J,\left(i_{1}, \ldots, i_{b}\right)}^{k: a}$ also vanishes on $\left(E_{\beta}, V_{m}\right)$. Note also that the decomposition (2.6) induces the decomposition

$$
R_{J, I}^{k ; a}=\sum_{1 \leq \alpha_{1}<\cdots<\alpha_{l-b} \leq l} \pm a_{i_{b+1}, \alpha_{1}} \ldots a_{i_{l}, \alpha_{l-b}} R_{J,\left(i_{\beta_{1}}, \ldots, i_{\beta_{b}}\right)}^{k ; a} .
$$

But as we have shown above, each of the relations $R_{J,\left(i_{\beta_{1}}, \ldots, i_{\beta_{b}}\right)}^{k ; a}$ vanishes on $\left(V_{l}, V_{m}\right)$. Hence so does $R_{J, I}^{k ; a}$.

Example 2.2. Let $\lambda=\omega_{1}+\omega_{n-1}$, i.e. $s=2, d_{1}=1, d_{2}=n-1$. Then the image of $\mathcal{F}^{a}(1, n-1)$ inside $\operatorname{Gr}(1, n) \times G r(n-1, n)$ is formed by all pairs $V_{1}, V_{2}$ such that $p r_{2, n-1} V_{1} \hookrightarrow V_{2}$. Since $p r_{2, n-1} V_{1} \hookrightarrow \operatorname{span}\left(v_{1}, v_{n}\right)$, the image of the embedding $\mathcal{F}^{a}(1, n-1) \hookrightarrow \mathbb{P}^{n-1} \times \mathbb{P}^{n-1}$ is defined by a single relation

$$
X_{1}^{a} X_{2, \ldots, n}^{a}+(-1)^{n-1} X_{n}^{a} X_{1, \ldots, n-1}^{a}=0
$$

which agrees with Example 1.2.

Corollary 2.1. Theorem 2.1 is true.

Corollary 2.2. Let $I^{1}, \ldots, I^{s}, I^{l} \subset\{1, \ldots, n\}$ be a collection of tuples such that the cardinality of $I^{l}$ is $d_{l}$. Then a point $p_{I^{1}} \times \cdots \times p_{I^{s}}$ belongs to $\mathcal{F}^{a}$ if and only if

$$
I^{l} \backslash\left\{d_{l}+1, \ldots, d_{l+1}\right\} \subset I^{l+1} .
$$

Example 2.3. Consider the case of the complete flags: $s=n-1, d_{l}=l$. Set $p r_{l}=p r_{l, l}$. Then the embedding of $\mathcal{F}^{a}$ into the product of Grassmannians is defined by the conditions

$$
\operatorname{pr}_{l+1} V_{l} \hookrightarrow V_{l+1}, l=1, \ldots, n-2
$$

and the conditions (2.7) read as $I^{l} \backslash\{l+1\} \subset I^{l+1}$ for $l=1, \ldots, n-2$.

2.3. Cells for $\mathcal{F}^{a}$. Recall that the cell decomposition for a Grassmannian is given by the $G^{a}$-orbits of the torus fixed points. However this is not true for the case of general $\mathcal{F}_{\lambda}^{a}$. Moreover, the number of $G^{a}$-orbits can be infinite. The simplest example is as follows.

Example 2.4. Let $n=4, \lambda=\omega_{1}+\omega_{3}$. Then $\mathcal{F}_{\lambda}^{a}$ is embedded into $\mathbb{P}^{3} \times \mathbb{P}^{3}$ (two Grassmannians for $\left.\mathfrak{s l}_{4}\right)$ with the coordinates $\left(x_{1}: x_{2}: x_{3}: x_{4}\right)$ and $\left(x_{123}: x_{124}\right.$ : $\left.x_{133}: x_{234}\right)$. The variety $\mathcal{F}_{\omega_{1}+\omega_{3}}^{a}$ is defined by a single relation $x_{1} x_{234}-x_{4} x_{123}=0$. Therefore, $\mathcal{F}_{\omega_{1}+\omega_{3}}^{a}$ contains the product $\mathbb{P}^{2} \times \mathbb{P}^{2}$ defined by $x_{1}=x_{123}=0$. We note that the subgroup $\mathbb{G}_{a}^{6}$ of $G^{a}$ acts trivially on this $\mathbb{P}^{2} \times \mathbb{P}^{2}$ (the PBW-degree in both $V_{\omega_{1}}$ and $V_{\omega_{3}}$ is at most one). Therefore, we are left with an action of the Borel subgroup. Let $w_{1}, w_{2}, w_{3}, w_{4}$ and $w_{123}, w_{124}, w_{134}, w_{234}$ be the standard bases for $V_{\omega_{1}}$ and $V_{\omega_{3}}$. 
The group $B$ acts on the span of $w_{2}, w_{3}, w_{4}$ (resp. on the span of $w_{124}, w_{134}, w_{234}$ ) as on the quotient of the vector representation (resp. the dual vector representation) by $\mathbb{C} w_{1}$ (resp. $\left.\mathbb{C} w_{123}\right)$. It is easy to see that the corresponding $B$-action on $\mathbb{P}^{2} \times \mathbb{P}^{2}$ has infinitely many orbits.

In the following proposition we describe the cell decomposition for $\mathcal{F}^{a}=\mathcal{F}^{a}\left(d_{1}, \ldots, d_{s}\right)$.

Proposition 2.3. Let $\mathbf{I}=\left(I^{1}, \ldots, I^{s}\right)$ be a set of sequences satisfying the condition (2.7). Then there exists a cell decomposition $\mathcal{F}^{a}=\sqcup_{\mathbf{I}} C_{\mathbf{I}}$, where

$$
C_{\mathbf{I}}=\left(G^{a} \cdot p_{I^{1}} \times \cdots \times G^{a} \cdot p_{I^{s}}\right) \cap \mathcal{F}^{a} .
$$

In other words, a cell is given by the intersection of the degenerate flag variety, embedded into the product of Grassmannians, with the product of the corresponding cells in $\operatorname{Gr}\left(d_{i}, n\right)$.

Proof. In Theorem 3.1 we compute the dimensions of $C_{\mathbf{I}}$. In the proof we construct explicitly the coordinates on $C_{\mathbf{I}}$ thus showing that $C_{\mathbf{I}}$ is a cell.

\section{The median Genocchi numbers}

3.1. Combinatorics. Let $h_{n}$ be the normalized Genocchi numbers of the second kind. They are also referred to as the normalized median Genocchi numbers. These numbers have several definitions (see [De, Du, Kr, S]). The first several $h_{n}$ 's are as follows: $1,2,7,38,295,3098$. We first briefly recall definitions of these numbers.

We start with the Dellac definition (see [De]). Consider a rectangle with $n$ columns and $2 n$ rows. It contains $n \times 2 n$ boxes labeled by pairs $(l, j)$, where $l=1, \ldots, n$ is the number of a column and $j=1, \ldots, 2 n$ is the number of a row. A Dellac configuration $D$ is a subset of boxes, subject to the following conditions:

- each column contains exactly two boxes from $D$,

- each row contains exactly one box from $D$,

- if the $(l, j)$-th box is in $D$, then $l \leq j \leq n+l$.

Let $D C_{n}$ be the set of such configurations. Then the number of elements in $D C_{n}$ is equal to $h_{n}$.

We list all Dellac's configurations for $n=3$. We specify boxes in a configuration by putting fat dots inside.
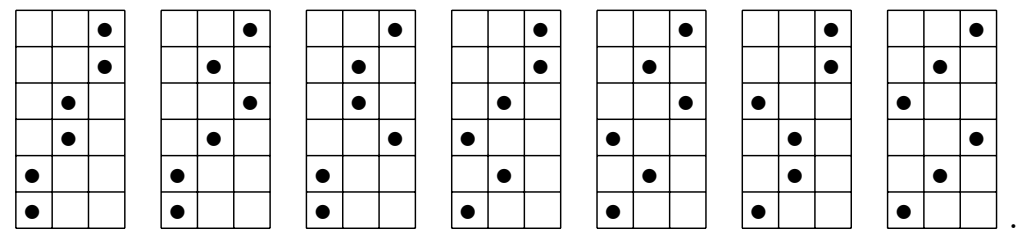
The Dellac definition is the earliest one, but the most well-known definition is via the Seidel triangle. The Seidel triangle is of the form

$\begin{array}{cccccc}1 & & & & \\ 1 & & & & \\ 1 & 1 & & & \\ 2 & 1 & & & \\ 2 & 3 & 3 & & \\ 8 & 6 & 3 & & \\ 8 & 14 & 17 & 17 & \\ 56 & 48 & 34 & 17 & \\ 56 & 104 & 138 & 155 & 155\end{array}$

By definition, the triangle is formed by the numbers $G_{k, n}$ ( $n$ is the number of a row and $k$ is the number of a column) with $n=1,2, \ldots$ and $1 \leq k \leq \frac{n+1}{2}$, subject to the relations $G_{1,1}=1$ and

$$
G_{k, 2 n}=\sum_{i \geq k} G_{i, 2 n-1}, G_{k, 2 n+1}=\sum_{i \leq k} G_{i, 2 n} .
$$

The numbers $G_{n, 2 n-1}$ are called the Genocchi numbers of the first kind and the numbers $G_{1,2 n}$ are called the Genocchi numbers of the second kind (or the median Genocchi numbers). Barsky $[\mathrm{Ba}]$ and then Dumont $[\mathrm{Du}]$ proved that the number $G_{1,2 n+2}$ is divisible by $2^{n}$. The normalized median Genocchi numbers $h_{n}$ are defined as the corresponding ratios: $h_{n}=G_{1,2 n+2} / 2^{n}$.

In $[\mathrm{Kr}]$ Kreweras suggested another description of the numbers $h_{n}$. Namely, a permutation $\sigma \in S_{2 n+2}$ is called a normalized Dumont permutation of the second kind if the following conditions are satisfied:

- $\sigma(k)<k$ if $k$ is even,

- $\sigma(k)>k$ if $k$ is odd,

- $\sigma^{-1}(2 k)<\sigma^{-1}(2 k+1)$ for $k=1, \ldots, n$.

The set of such permutations is denoted by $P D 2 N_{n}$ (P for permutations, D for Dumont, 2 for the second kind and $\mathrm{N}$ for normalized). According to Kreweras, the number of elements of $P D 2 N_{n}$ is equal to $h_{n}$. In Proposition 3.2 we show that the definitions of Dellac and Kreweras are equivalent (this seems to be known to expert see $[G, S]$, but we were not able to find a proof in the literature).

In the following proposition we show that the conditions from Example 2.3 give rise to a new definition of the numbers $h_{n}$.

Proposition 3.1. The number of tuples $I^{1}, \ldots, I^{n-1}$, with $I^{l} \subset\{1, \ldots, n\}, \# I^{l}=l$ subject to the condition

$$
I^{l-1} \backslash\{l\} \subset I^{l}, l=2, \ldots, n-1
$$

is equal to $h_{n}$.

Proof. Let $\bar{h}_{n}$ be the number of tuples as above. We compare $\bar{h}_{n}$ with the Dellac definition of $h_{n}$. Given a set $I^{1}, \ldots, I^{n-1}$ subject to the condition (3.2), we construct the corresponding Dellac's configuration $D$ and then prove that this map is one-to-one. The rule is as follows. Let us explain what are the boxes of $D$ in the $l$ th column. 
First, suppose $l \notin I^{l-1}$. Then because of the condition (3.2) the difference $I^{l} \backslash I^{l-1}$ contains exactly one number $j$. There are two cases:

- If $j>l$, then $D$ contains boxes $(l, l)$ and $(l, j)$.

- If $j \leq l$, then $D$ contains boxes $(l, l)$ and $(l, j+n)$.

Now, suppose $l \in I^{l-1}$. Then either $l \in I^{l}$, or $L \notin I^{l}$. If $l \in I^{l}$, then $I^{l} \backslash I^{l-1}$ contains exactly one number $j$. There are two cases:

- If $j>l$, then $D$ contains boxes $(l, l+n)$ and $(l, j)$.

- If $j \leq l$, then $D$ contains boxes $(l, l+n)$ and $(l, j+n)$.

Finally, let $l \in I^{l-1}$ and $l \notin I^{l}$. Then $I^{l} \backslash I^{l-1}$ contains exactly two numbers $j_{1}$ and $j_{2}$. There are four variants:

- If $j_{1}>l$ and $j_{2}>l$, then $D$ contains boxes $\left(l, j_{1}\right)$ and $\left(l, j_{2}\right)$.

- If $j_{1}>l$ and $j_{2} \leq l$, then $D$ contains boxes $\left(l, j_{1}\right)$ and $\left(l, n+j_{2}\right)$.

- If $j_{1} \leq l$ and $j_{2}>l$, then $D$ contains boxes $\left(l, j_{1}+n\right)$ and $\left(l, j_{2}\right)$.

- If $j_{1} \leq l$ and $j_{2} \leq l$, then $D$ contains boxes $\left(l, j_{1}+n\right)$ and $\left(l, j_{2}+n\right)$.

This rule explains how to pick boxes in columns from 1 to $n-1$. To complete the configuration we simply pick two boxes in the last column in the unique way to make $D$ a configuration.

In order to prove that this map is a bijection, we construct the inverse map. Let $D$ be a Dellac configuration. We define $I^{l}$ inductively. First, let $l=1$. Then the box $(1,1)$ necessarily belongs to $D$. Let $j>1$ and $D$ contains $(1, j)$. Then if $j=n+1$, then $I^{1}=(1)$. Otherwise $I^{1}=(j)$.

Now assume that $I^{l-1}$ is already defined. First, suppose that the $(l, l)$-th box belongs to $D$. Then there exists one more box $(l, j)$ in $D$ with $n+l \geq j>l$. If $j \leq n$ we set $I^{l}=I^{l-1} \cup\{j\}$. Otherwise, we set $I^{l}=I^{l-1} \cup\{j-n\}$. Second, suppose that the $(l, l)$-th box does not belong to $D$. Since the $l$ th row of $D$ contains exactly one box, there exists $l_{1}<l$ such that the $\left(l_{1}, l\right)$-th box belongs to $D$. Therefore, $l \subset I^{l-1}$. There exist exactly two boxes $\left(l, j_{1}\right)$ and $\left(l, j_{2}\right)$ in $D$ in the $l$ th column. Then we set $I^{l}=I^{l-1} \backslash\{l\} \cup\left\{\bar{j}_{1}, \bar{j}_{2}\right\}$, where $\bar{j}=j$, if $j \leq n$ and $\bar{j}=j-n$ otherwise.

Example 3.1. Let $n=3$. The pairs $I^{1}, I^{2}$, corresponding to the Dellac configurations (3.1) are as follows (the order is the same as on picture (3.1)):

$$
\begin{gathered}
\{(2),(13)\}, \quad\{(2),(23)\}, \quad\{(2),(12)\}, \quad\{(3),(13)\}, \\
\{(3),(23)\}, \quad\{(1),(13)\}, \quad\{(1),(12)\} .
\end{gathered}
$$

We now compare the definitions by Dellac and by Kreweras.

Proposition 3.2. The number of elements in $P D 2 N_{n}$ is equal to the number of elements in $D C_{n}$.

Proof. We construct a bijection $A: P D 2 N_{n} \rightarrow D C_{n}$. Let $\sigma \in P D 2 N_{n}$. We determine the boxes in the $k$ th column of $A(\sigma)$ using the values of $\sigma^{-1}(2 k)$ and $\sigma^{-1}(2 k+1)$.

Let us start with $k=1$. We note that $\sigma(2)=1, \sigma(4)$ is equal to 2 or to 3 . In addition, $\sigma^{-1}(2)=1$ or 4 and the possible values of $\sigma^{-1}(3)$ are $4,6, \ldots, 2 n+2$. Therefore, all possible values of the pair $\left(\sigma^{-1}(2), \sigma^{-1}(3)\right)$ are as follows:

$$
(1,4),(4,6),(4,8), \ldots,(4,2 n+2) \text {. }
$$


If the first possibility occurs, then by definition the first column of $A(\sigma)$ contains boxes $(1,1)$ (as any Dellac's configuration) and $(1, n+1)$. If $\sigma^{-1}(2)=4$ and $\sigma^{-1}(3)=2 l+2$, then the first column of $A(\sigma)$ contains boxes $(1,1)$ and $(1, l)$.

Now let us consider the case $k=n$. We note that $\sigma(2 n+1)=2 n, \sigma(2 n-1)$ is equal to $2 n$ or to $2 n+1$. In addition, $\sigma^{-1}(2 n+1)=2 n+2$ or $2 n-1$ and the possible values of $\sigma^{-1}(2 n)$ are $1,3, \ldots, 2 n-1$. Therefore, all possible values of the pair $\left(\sigma^{-1}(2 n), \sigma^{-1}(2 n+1)\right)$ are as follows:

$$
(2 n-1,2 n+2),(1,2 n-1),(3,2 n-1), \ldots,(2 n-3,2 n-1) .
$$

If the first possibility occurs, then by definition the $n$th column of $A(\sigma)$ contains boxes $(n, 2 n)$ (as any Dellac's configuration) and $(n, n)$. If

$$
\left(\sigma^{-1}(2 n), \sigma^{-1}(2 n+1)\right)=(2 l-1,2 n-1),
$$

then the first column of $A(\sigma)$ contains boxes $(n, 2 n)$ and $(n, n+l)$.

Finally, take $k=2, \ldots, n-1$. We note that the possible values of $\sigma^{-1}(2 k)$ are $1,3, \ldots, 2 k-1,2 k+2, \ldots, 2 n$. Also, the possible values of $\sigma^{-1}(2 k+1)$ are $3,5, \ldots, 2 k-$ $1,2 k+2, \ldots, 2 n, 2 n+2$. We now define the $k$ th column of $A(\sigma)$ as follows:

(i) If the pair $\left(\sigma^{-1}(2 k), \sigma^{-1}(2 k+1)\right)$ contains $2 l-1, l=1, \ldots, k$, then the $k$ th column of $A(\sigma)$ contains a box $(k, n+l)$.

(ii) If the pair $\left(\sigma^{-1}(2 k), \sigma^{-1}(2 k+1)\right)$ contains $2 l+2, l=k, \ldots, n$, then the $k$ th column of $A(\sigma)$ contains a box $(k, l)$.

We note that $A(\sigma) \in D C_{n}$. In fact, by definition any column of $A(\sigma)$ contains exactly two boxes and every row contains exactly one box (this follows from the definition above and because $\sigma$ is one-to-one). In order to prove that $A$ is a bijection it suffices to note that formulas (i) and (ii) allow to construct explicitly the map $A^{-1}$.

Example 3.2. Let $n=3$. The elements of $P D 2 N_{3}$ corresponding to the Dellac configurations on picture (3.1) are as follows (the order is the same as on picture $(3.1))$ :

$$
\begin{gathered}
\text { (41627385), (61427385), (41526387), (41627583), } \\
\text { (61427583), (21637485), (21436587). }
\end{gathered}
$$

We recall that the main ingredient for the Kreweras construction of $P D 2 N_{n}$ is the following triangle:

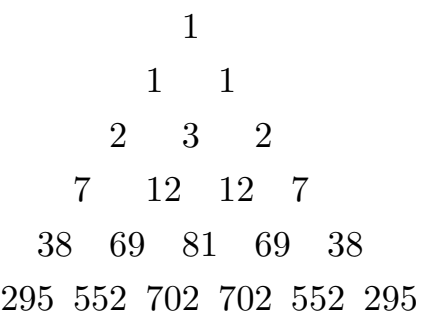

The rule is as follows: denote the numbers in the $n$th line by $h_{n, 1}, \ldots, h_{n, n}$. For example, $h_{4,2}=12$. Then the Kreweras triangle is defined by

$$
\begin{aligned}
& h_{n, 1}=h_{n-1,1}+\cdots+h_{n-1, n-1}, h_{n, 2}=2 h_{n, 1}-h_{n-1,1}, \\
& h_{n, k}=2 h_{n, k-1}-h_{n, k-2}-h_{n-1, k-2}-h_{n-1, k-1}, k \geq 3 .
\end{aligned}
$$


Kreweras proved that $h_{n+1,1}$ is the $n$th Genocchi number $h_{n}$ and in general $h_{n+1, k}$ is the number of the normalized Dumont permutations $\sigma \in S_{2 n+2}$ of the second kind such that $\sigma(1)=2 k$. The following is an immediate corollary from the explicit bijections above.

Corollary 3.1. The number of the Dellac configurations $D \in D C_{n}$ such that $\min \{i$ : $(i, n+1) \in D\}=k$ is equal to $h_{n, k}$. The number of tuples $I^{1}, \ldots, I^{n-1}$ subject to the condition $I^{l-1} \backslash\{l\} \subset I^{l}$ with an extra condition $\min \left\{j: 1 \in I^{j}\right\}=k$ is equal to $h_{n, k}$.

3.2. The Poincaré polynomials. For a tuple $\mathbf{I}=\left(I^{1}, \ldots, I^{n-1}\right)$ subject to the relation $I^{l-1} \backslash\{l\} \subset I^{l}$ we denote by $D_{\mathbf{I}}$ the corresponding Dellac configuration. For a Dellac configuration $D \in D C_{n}$ we define the length $l(D)$ of $D$ as the number of pairs $\left(l_{1}, j_{1}\right),\left(l_{2}, j_{2}\right)$ such that the boxes $\left(l_{1}, j_{1}\right)$ and $\left(l_{2}, j_{2}\right)$ are both in $D$ and $l_{1}<l_{2}$, $j_{1}>j_{2}$. We call such a pair of boxes $\left(l_{1}, j_{1}\right),\left(l_{2}, j_{2}\right)$ a disorder. This definition resembles the definition of the length of a permutation. We note that in the classical case the dimension of a cell attached to a permutation $\sigma$ in a flag variety is equal to the number of pairs $j_{1}<j_{2}$ such that $\sigma\left(j_{1}\right)>\sigma\left(j_{2}\right)$ (which equals to the length of $\sigma$ ).

Theorem 3.1. The dimension of a cell $C_{\mathbf{I}}$ is equal to $l\left(D_{\mathbf{I}}\right)$.

Proof. We prove the dimension formula by constructing explicitly the coordinates on the cell $C_{\mathbf{I}}$. Let

$$
\mathbf{I}=\left(I^{1}, \ldots, I^{n-1}\right), I^{d}=\left(i_{1}^{d}<\cdots<i_{d}^{d}\right) .
$$

Recall the description of the cells $C_{I^{d}} \subset G r(d, n)$ from Proposition 2.1. Using this description we construct the coordinates on $C_{\mathbf{I}}$ inductively on $d$. Let $\left(V_{1}, \ldots, V_{n-1}\right) \in$ $C_{\mathbf{I}}$. For a number $k$ we set $[k]_{+}=k$ if $k>0$ and $[k]_{+}=k+n$ if $k \leq 0$.

We start with $d=1$. An element $V_{1} \in C_{I^{1}}$ is of the form $\mathbb{C} e_{1}^{1}$ with

$$
e_{1}^{1}=v_{i_{1}^{1}}+a_{1}^{1} v_{\left[i_{1}^{1}-1\right]_{+}}+\cdots+a_{\left[i_{1}^{1}-1\right]_{+}-1}^{1} v_{2}
$$

(see Remark 2.1). We state that $\left[i_{1}^{1}-1\right]_{+}-1$ (which is exactly the number of the degrees of freedom we have so far) is exactly the number of boxes $(l, j) \in D_{\mathbf{I}}$ such that $l>1$ and $j<i_{1}^{1}$ (note that the box $(1,1)$ is necessarily in $D_{\mathbf{I}}$, but it does not add anything to the length of $D_{\mathbf{I}}$, since for any $(l . j) \in D_{\mathbf{I}}$ with $l>1$ we have $\left.j>1\right)$. In fact, the first column of $D_{\mathbf{I}}$ contains boxes in the first row and in the $\left(\left[i_{1}^{1}-1\right]_{+}+1\right)$-st row (see the proof of Proposition 3.1). Since any row of $D_{\mathbf{I}}$ contains exactly one box, the rows number $2, \ldots,\left[i_{1}^{1}-1\right]_{+}$are occupied by boxes in the columns from 2 to $n$. Therefore, the box $\left(1,\left[i_{1}^{1}-1\right]_{+}+1\right)$ produces exactly $\left[i_{1}^{1}-1\right]_{+}-1$ disorders.

The second step is to construct the coordinates on those subspaces from $C_{I^{2}}$ which contain $p r_{2} V_{1}$. There are two possibilities: either $i_{1}^{1}=2$ or $i_{1}^{1} \neq 2$. In the first case the condition $p r_{2} V_{1} \hookrightarrow V_{2}$ is empty. Therefore, we have to choose two basis vectors $e_{1}^{2}, e_{2}^{2}$ of $V_{2} \in C_{I^{2}}$, with the coordinates

$$
\begin{aligned}
& e_{1}^{2}=v_{i_{1}^{2}}+a_{1}^{1} v_{\left[i_{1}^{2}-1\right]_{+}}+\cdots+a_{\left[i_{1}^{2}-2\right]_{+}-1}^{1} v_{3}, \\
& e_{2}^{2}=v_{i_{2}^{2}}+a_{1}^{2} v_{\left[i_{2}^{2}-1\right]_{+}}+\cdots+a_{\left[i_{2}^{2}-2\right]_{+}-2}^{2} v_{3} .
\end{aligned}
$$

We note that the number of coefficients of $e_{2}^{2}$ is $\left[i_{2}^{2}-2\right]_{+}-2$, because $i_{1}^{2}<i_{2}^{2}$ and hence adding appropriately normalized vector $e_{1}^{2}$ one can vanish the coefficient of $e_{2}^{2}$ in front of $v_{i_{1}^{2}}$. We note that since $i_{1}^{1}=2$, the second column of $D_{\mathbf{I}}$ contains boxes in the rows $\left(\left[i_{1}^{2}-2\right]_{+}+2\right)$ and $\left(\left[i_{1}^{2}-2\right]_{+}+2\right)$ (see the proof of Proposition 3.1). We state that 
$\left[i_{1}^{2}-2\right]_{+}-1+\left[i_{2}^{2}-2\right]_{+}-2$ (the number of degrees of freedom we have fixing the vectors $e_{1}^{2}$ and $\left.e_{2}^{2}\right)$ is exactly the number of boxes in the columns $3,4, \ldots, n$, having disorders with boxes in the second column. In fact, each row from 3 to $\left[i_{1}^{2}-2\right]_{+}-1$ contains one box in the columns 3 and greater (recall $i_{1}^{1}=2$ ). This produces $\left[i_{1}^{2}-2\right]_{+}-1$ disorders with the box $\left(2,\left[i_{1}^{2}-2\right]_{+}-1\right)$. Similarly, we obtain $\left[i_{2}^{2}-2\right]_{+}-2$ disorders with the second box in the second column.

Now assume $i_{1}^{1} \neq 2$. Then the space $p r_{2} V_{1}$ is nontrivial and spanned by a single vector $e_{1}^{2}=p r_{2} e_{1}^{1}$. Therefore in order to specify $V_{2}$ we need to fix one more vector $e_{2}^{2}$ such that $\operatorname{span}\left(e_{1}^{2}, e_{2}^{2}\right) \in C_{I^{2}}$. Recall that since $i_{1}^{1} \neq 2$ we have $I^{2} \backslash I^{1}=\{j\}$. Also, the second column of $D_{\mathbf{I}}$ contains boxes in the second row and in the row number $[j-2]_{+}+2$ (see the proof of Proposition 3.1). The box $(2,2)$ does not produce any disorder with boxes in the columns greater than 2 . As for the box $\left(2,[j-2]_{+}+2\right)$, the number of disorders it produces is equal to the number of degrees of freedom of choosing the vector $e_{2}^{2}$ (the argument is very similar to the ones above in the case $\left.i_{1}^{1}=2\right)$.

Now let us consider the general induction step. Assume that we have already computed the number of degrees of freedom while fixing the subspaces $V_{1}, \ldots, V_{d-1}$. Our goal is to show that the number of degrees of freedom of $V_{d}$ is equal to the number of disorders produced by the boxes in the $d$ 'th column with the boxes in columns $l$ with $l>d$. As in the previous case, one has to consider two cases: $d \in I^{d-1}$ and $d \notin I^{d-1}$. The proof is very similar to the one in the case $d=2$ and we omit it.

Corollary 3.2. The Poincaré polynomial $P_{n}(t)=P_{\mathcal{F}^{a}}(t)$ is given by

$$
P_{n}(t)=\sum_{D \in D C_{n}} t^{2 l(D)} .
$$

Let $q=t^{2}$. Then $P_{n}$ are polynomials in $q$ with $P_{n}(1)=h_{n}$. Thus the Poincaré polynomials of the degenerate flag varieties provide a natural $q$-version of the normalized median Genocchi numbers (it would be interesting to compare our $q$-version with the one in $[\mathrm{HZ}])$.

Example 3.3. The first four polynomials $P_{n}(q)$ are as follows:

$$
\begin{gathered}
P_{1}(q)=1, \quad P_{2}(q)=1+q, \\
P_{3}(q)=1+2 q+3 q^{2}+q^{3}, \\
P_{4}(q)=1+3 q+7 q^{2}+10 q^{3}+10 q^{4}+6 q^{5}+q^{6} .
\end{gathered}
$$

\section{Acknowledgments}

This work was partially supported by the Russian President Grant MK-281.2009.1, RFBR Grant 09-01-00058, by grant Scientific Schools 6501.2010.2 and by the Dynasty Foundation.

\section{References}

[A] I. Arzhantsev, Flag varieties as equivariant compactifications of $\mathbb{G}_{a}^{n}$, arXiv:1003.2358.

[AS] I. Arzhantsev and E. Sharoiko, Hassett-Tschinkel correspondence: modality and projective hypersurfaces, arXiv:0912.1474.

[Ba] D. Barsky, Congruences pour les nombres de Genocchi de 2e espèce, Groupe d'étude d'Analyse ultramétrique, 8e année, no. 34, 1980/81, 13 pp. 
[De] H. Dellac, Problem 1735, L'Intermédiaire des Mathématiciens 7 (1900), 9-10.

[Du] D. Dumont, Interprétations combinatoires des nombres de Genocchi, Duke Math. J. 41 (1974), 305-318.

[DR] D. Dumont and A. Randrianarivony, Dérangements et nombres de Genocchi, Discrete Math. 132 (1994), 37-49.

[DZ] D. Dumont and J. Zeng, Further results on Euler and Genocchi numbers, Aequationes Mathemicae 47 (1994), 31-42.

[Fe1] E. Feigin, The PBW filtration, Represent. Theory 13 (2009), 165-181.

$[\mathrm{Fe} 2]$ E. Feigin, The PBW filtration, Demazure modules and toroidal current algebras, SIGMA, 4 (2008), 070, $21 \mathrm{p}$.

[Fe3] E. Feigin, $\mathbb{G}_{a}^{M}$ degeneration of flag varieties, arXiv:1007.0646,

[Fu W. Fulton, Young tableaux, with applications to representation theory and geometry, Cambridge University Press (1997).

[FFoL1] E. Feigin, G. Fourier and P. Littelmann, $P B W$ filtration and bases for irreducible modules in type $A_{n}$, arXiv:1002.0674.

[FFoL2] E. Feigin, G. Fourier and P. Littelmann, PBW filtration and bases for symplectic Lie algebras, arXiv:1010.2321.

[G] I. Gessel, Applications of the classical umbral calculus, Algebra Universalis 49 (2003), 397-434.

[HZ] G.-N. Han and J. Zeng, On a q-sequence that generalizes the median Genocchi numbers, Ann. Sci. Math. Québec 23 (1999), 63-72.

[HT] B. Hassett and Yu. Tschinkel, Geometry of equivariant compactifications of $\mathbb{G}_{a}^{n}$, Int. Math. Res. Not. 20 (1999), 1211-1230.

[Kr] G. Kreweras, Sur les permutations comptées par les nombres de Genocchi de 1-ière et 2-ième espèce, Europ. J. Combinatorics 18 (1997), 49-58.

[K1] S. Kumar, Kac-Moody groups, their flag varieties and representation theory, Progress in Mathematics 204, Birkhauser, Boston, 606, 2002.

[K2] S. Kumar, The nil Hecke ring and singularity of Schubert varieties, Inventiones Math. 123, (1996), 471-506.

[S] N. J. A. Sloane, Sequence A000366, The on-line encyclopedia of integer sequences, http://oeis.org.

[Vien] G. Viennot, Interprétations combinatoires des nombres d'Euler et de Genocchi, Seminar on Number Theory, 1981/1982, No. 11, 94 pp., Univ. Bordeaux I, Talence, 1982.

Department of Mathematics, National Research University Higher School of Economics, Vavilova street 7, Moscow 117312, Russia

Tamm Theory Division, Lebedev Physics Institute, Moscow 117924, Russia

E-mail address: evgfeig@gmail.com 